\title{
The Improvement of the Hepatic Histological Findings in a Patient with Non-alcoholic Steatohepatitis with Type 2 Diabetes after the Administration of the Sodium-glucose Cotransporter 2 Inhibitor Ipragliflozin
}

\author{
Akihiko Takeda ${ }^{1}$, Aya Irahara $^{1}$, Atsuko Nakano ${ }^{1}$, Emi Takata $^{1}$, Yuko Koketsu ${ }^{1}$, \\ Kunie Kimata ${ }^{1}$, Eri Senda ${ }^{2}$, Hajime Yamada ${ }^{2}$, Kazuhito Ichikawa ${ }^{3}$, \\ Takahiro Fujimori $^{3}$ and Yoshio Sumida ${ }^{4}$
}

\begin{abstract}
:
The patient was a 67-year-old woman with type 2 diabetes and non-alcoholic steatohepatitis (NASH). The administration of the sodium-glucose cotransporter 2 (SGLT2) inhibitor, ipragliflozin improved her liver dysfunction clinically and histologically. The serum alanine aminotransferase (ALT) and ferritin levels decreased to normal limits after treatment for four months. Type IV collagen and hyaluronic acid, both of which were serum fibrotic markers, decreased after treatment. Ultrasonography and computed tomography showed a decrease in the fat deposits in her liver. Her liver sample showed marked improvement, especially in steatosis, inflammation, and ballooning. The SGLT2 inhibitor ipragliflozin may be useful as a specific therapeutic drug for NASH.
\end{abstract}

Key words: hyaluronic acid, ipragliflozin, NASH, type IV collagen, type 2 diabetes

(Intern Med 56: 2739-2744, 2017)

(DOI: 10.2169/internalmedicine.8754-16)

\section{Introduction}

Non-alcoholic steatohepatitis (NASH) or non-alcoholic fatty liver disease (NAFLD) are reaching epidemic proportions in patients with type 2 diabetes. Patients with NASH are at a high risk of death from hepatocellular carcinoma and cardiovascular disease. However, there are no specific therapeutic drugs for NASH, so managing this disease is difficult. Sodium-glucose cotransporter 2 (SGLT2) inhibitors are new drugs used in the management of type 2 diabetes, that control hyperglycemia by the inhibition of proximal tube glucose reabsorption. In animal models of NASH, SGLT2 inhibitors have shown unique anti-fibrotic properties. In patients with diabetes, the levels of plasma aminotransferases decrease during treatment with SGLT2 inhibitors, but histological findings of improvement have not been reported. We herein report the first case of NASH with type 2 diabetes whose hepatic histological findings were improved by the administration of the SGLT2 inhibitor, ipragliflozin.

\section{Case Report}

A 67-year-old woman with type 2 diabetes was known to have liver dysfunction for a long time. She had been diagnosed with type 2 diabetes in 1998, and ultrasonography had shown a fatty liver (data not shown) at that time. She had no drinking habit, hepatitis virus infection, or autoimmune disease (Table 1). She was therefore clinically diagnosed with NASH, because her platelet count was under $15.3 \times 10^{4} / \mathrm{mL}$ (1). She was not obese [body mass index (BMI) $23.3 \mathrm{~kg} / \mathrm{m}^{2}$ ] but had a history of obesity (maximum

\footnotetext{
${ }^{1}$ Department of Diabetic and Metabolic Medicine, Shinko Hospital, Japan, ${ }^{2}$ Department of Gastroenterology, Shinko Hospital, Japan, ${ }^{3}$ Department of Pathology of Shinko Hospital, Japan and ${ }^{4}$ Division of Hepatology and Pancreatology, Department of Internal Medicine, Aichi Medical University, Japan

Received: December 22, 2016; Accepted: February 28, 2017; Advance Publication by J-STAGE: September 15, 2017

Correspondence to Dr. Akihiko Takeda, a-takeda@shinkohp.or.jp
} 
Table 1. Laboratory Findings Just before Administration of Ipragliflozin.

\begin{tabular}{|c|c|c|c|c|c|}
\hline WBC & $5,400 / \mu \mathrm{L}$ & $\mathrm{TP}$ & $8.0 \mathrm{~g} / \mathrm{dL}$ & T-Cho & $184 \mathrm{mg} / \mathrm{dL}$ \\
\hline $\mathrm{RBC}$ & $464 \times 10^{4} / \mu \mathrm{L}$ & $\mathrm{Alb}$ & $4.6 \mathrm{~g} / \mathrm{dL}$ & $\mathrm{TG}$ & $259 \mathrm{mg} / \mathrm{dL}$ \\
\hline $\mathrm{Hb}$ & $13.0 \mathrm{~g} / \mathrm{dL}$ & AST & $106 \mathrm{U} / \mathrm{L}$ & HDL-C & $44 \mathrm{mg} / \mathrm{dL}$ \\
\hline $\mathrm{Ht}$ & $38.5 \%$ & ALT & $97 \mathrm{U} / \mathrm{L}$ & LDL-C & $108 \mathrm{mg} / \mathrm{dL}$ \\
\hline \multirow[t]{2}{*}{ Plt } & $11.9 \times 10^{4} / \mu \mathrm{L}$ & ALP & $246 \mathrm{U} / \mathrm{L}$ & PG & $226 \mathrm{mg} / \mathrm{dL}$ \\
\hline & & $\mathrm{TB}$ & $0.6 \mathrm{mg} / \mathrm{dL}$ & $\mathrm{HbA} 1 \mathrm{c}$ & $8.5 \%$ \\
\hline HBs-Ag & $(-)$ & LDH & $261 \mathrm{U} / \mathrm{L}$ & IRI & $22.0 \mu \mathrm{U} / \mathrm{mL}$ \\
\hline $\mathrm{HCV}-\mathrm{Ab}$ & $(-)$ & $\gamma$-GPT & $86 \mathrm{U} / \mathrm{L}$ & TSH & $2.102 \mu \mathrm{U} / \mathrm{mL}$ \\
\hline ANA & $<x 40$ & $\mathrm{ChE}$ & $386 \mathrm{U} / \mathrm{L}$ & fT4 & $1.09 \mathrm{ng} / \mathrm{dL}$ \\
\hline \multirow[t]{2}{*}{ AMA } & $<\times 20$ & $\mathrm{CK}$ & $237 \mathrm{U} / \mathrm{L}$ & & \\
\hline & & Amy & $58 \mathrm{U} / \mathrm{L}$ & & \\
\hline AFP & $3 \mathrm{ng} / \mathrm{mL}$ & BUN & $14.6 \mathrm{mg} / \mathrm{dL}$ & & \\
\hline \multirow[t]{6}{*}{ PIVKA II } & $27 \mathrm{mAU} / \mathrm{mL}$ & sCre & $0.91 \mathrm{mg} / \mathrm{dL}$ & & \\
\hline & & UA & $5.2 \mathrm{mg} / \mathrm{dL}$ & & \\
\hline & & $\mathrm{Na}$ & $140 \mathrm{mEq} / \mathrm{L}$ & & \\
\hline & & K & $4.0 \mathrm{mEq} / \mathrm{L}$ & & \\
\hline & & $\mathrm{Cl}$ & $102 \mathrm{mEq} / \mathrm{L}$ & & \\
\hline & & CRP & $0.07 \mathrm{mg} / \mathrm{dL}$ & & \\
\hline
\end{tabular}

ANA: anti-nuclear antibody, AMA: anti-mitochondria antibody

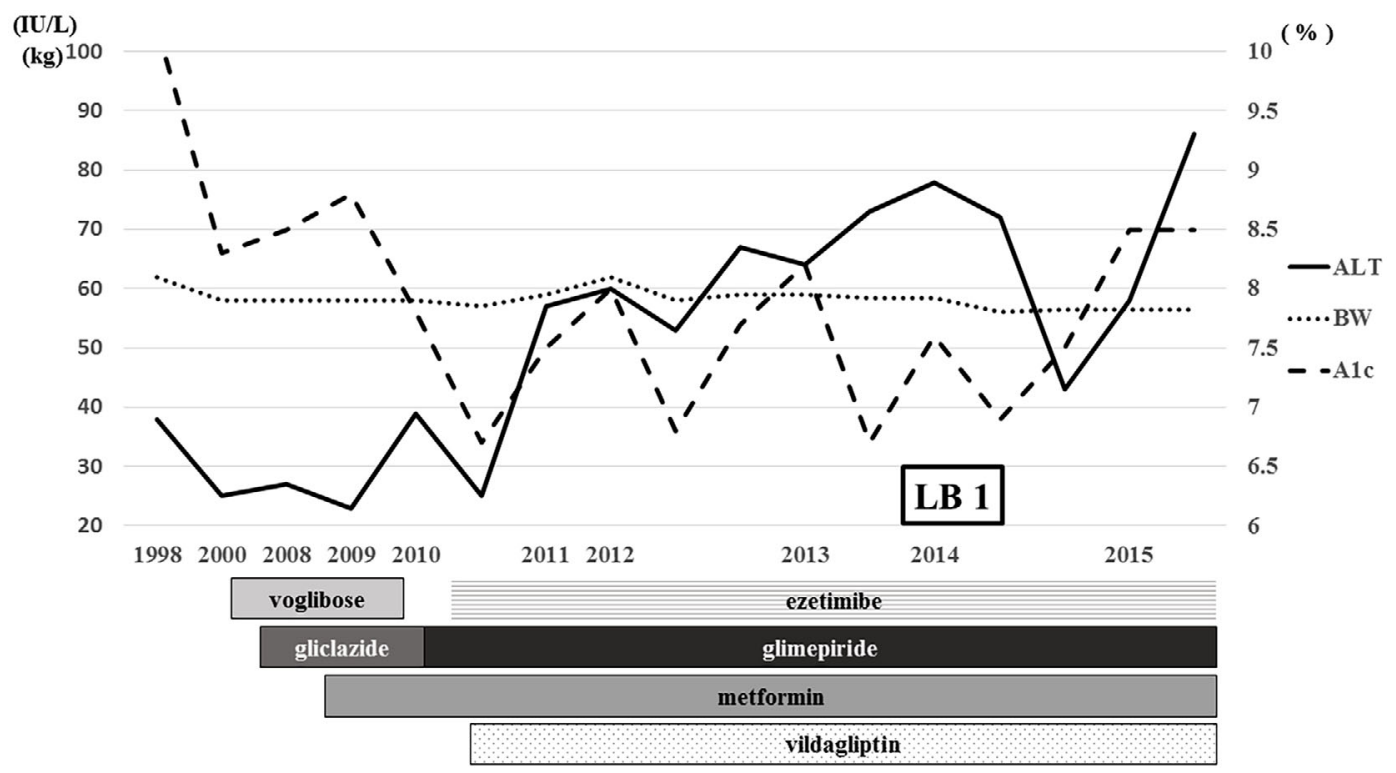

Figure 1. Clinical course before the administration of ipragliflozin. ALT: alanine aminotransferase, BW: body weight, A1c: glycated hemoglobin, LB: liver biopsy

BMI $\left.27.0 \mathrm{~kg} / \mathrm{m}^{2}\right)$. Regarding her liver data, her alanine aminotransferase (ALT) value had been 40-80 IU/L since 2011, and her glycated hemoglobin (HbA1c) levels ranged from 6-8\%. She was already receiving oral antidiabetic drugs and the lipid-lowering agent, ezetimibe (Fig. 1).

She suffered from acute cholecystitis and underwent laparoscopic surgery in 2014. The surgeon obtained a liver sample during gallbladder resection after receiving informed consent. She was then histologically diagnosed with NASH for the first time (Fig. 2). Her NAFLD activity score (NAS) was 6 (steatosis 2, inflammation 2, ballooning 2), and her fibrosis score was 4 (2). After surgery, her liver data showed temporary improvement but worsened gradually again, and her $\mathrm{HbA1c}$ level increased again to $8.5 \%$ (Fig. 1). She started oral administration of ipragliflozin at $50 \mathrm{mg} /$ day in September 2015.

After treatment for four months, her HbA1c level gradually decreased to $8 \%$, and her ALT level decreased dramatically to normal limits (Fig. 3). Her body weight also decreased to $55.2 \mathrm{~kg}$ (-1.4 $\mathrm{kg}$ from baseline). Ultrasonography showed a decrease in the fat deposits in her liver (data not shown), and her liver/spleen ratio calculated by computed tomography increased from 1.02 to 1.10 (Fig. 4). Ferritin, a serum inflammatory marker, decreased from $132.4 \mathrm{ng} / \mathrm{mL}$ to $38.6 \mathrm{ng} / \mathrm{mL}$. Type IV collagen and hyaluronic acid, both of which were serum fibrotic markers, decreased from $271 \mathrm{ng} /$ $\mathrm{mL}$ to $207 \mathrm{ng} / \mathrm{mL}$ and from $596 \mathrm{ng} / \mathrm{mL}$ to $517 \mathrm{ng} / \mathrm{mL}$, respectively (Table 2). 


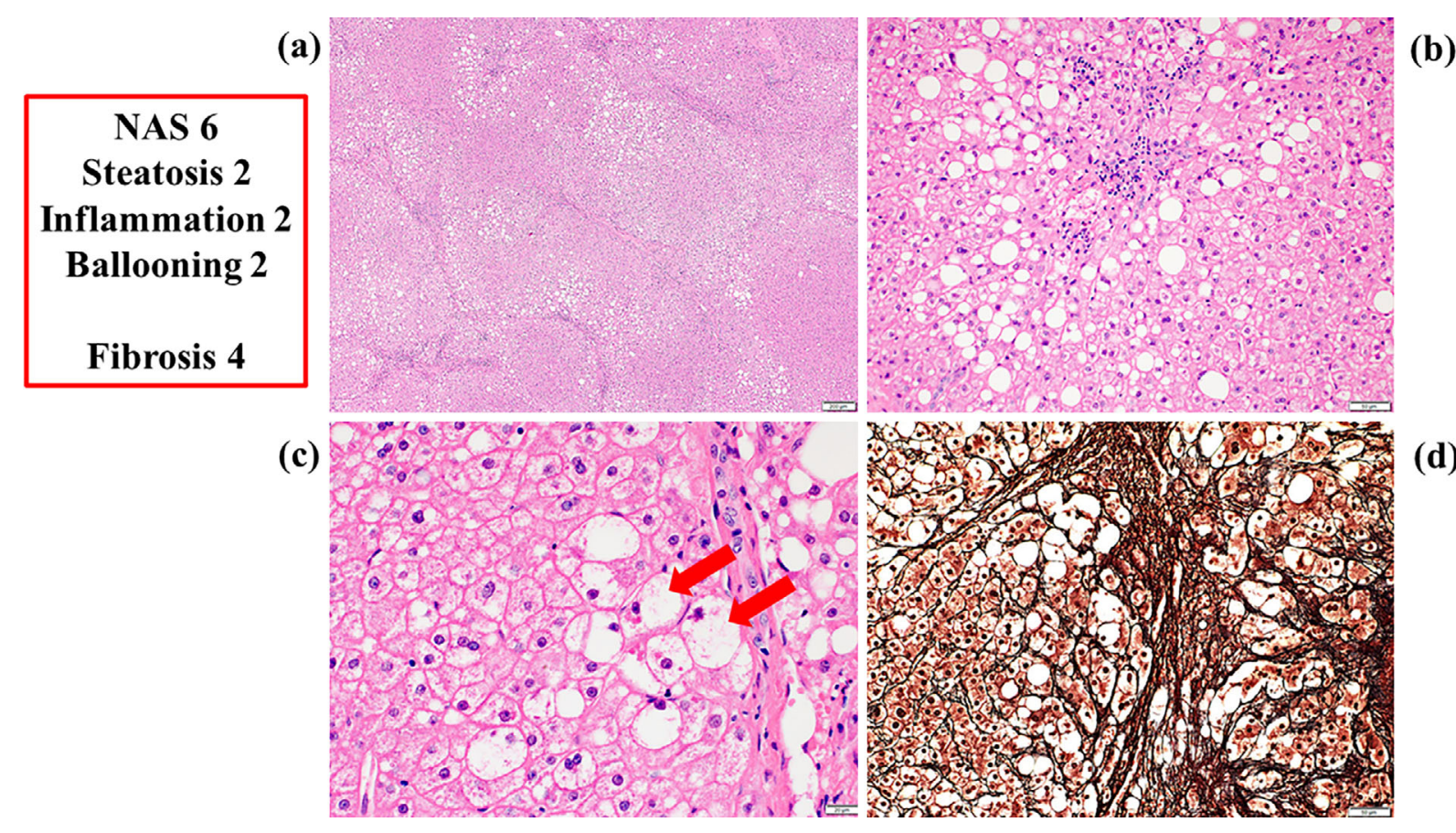

Figure 2. Histological findings of liver samples before the administration of ipragliflozin. a: An overview of the liver sample [Hematoxylin and Eosin (H\&E) staining, 50×]. b: Fat droplets and inflammatory neutrophil aggregations (H\&E staining, 200x). c: Hepatocellular ballooning (arrow) (H\&E staining, 500x). d: Interstitial fibrosis (silver staining, 200x). NAS: non-alcoholic fatty liver disease activity score

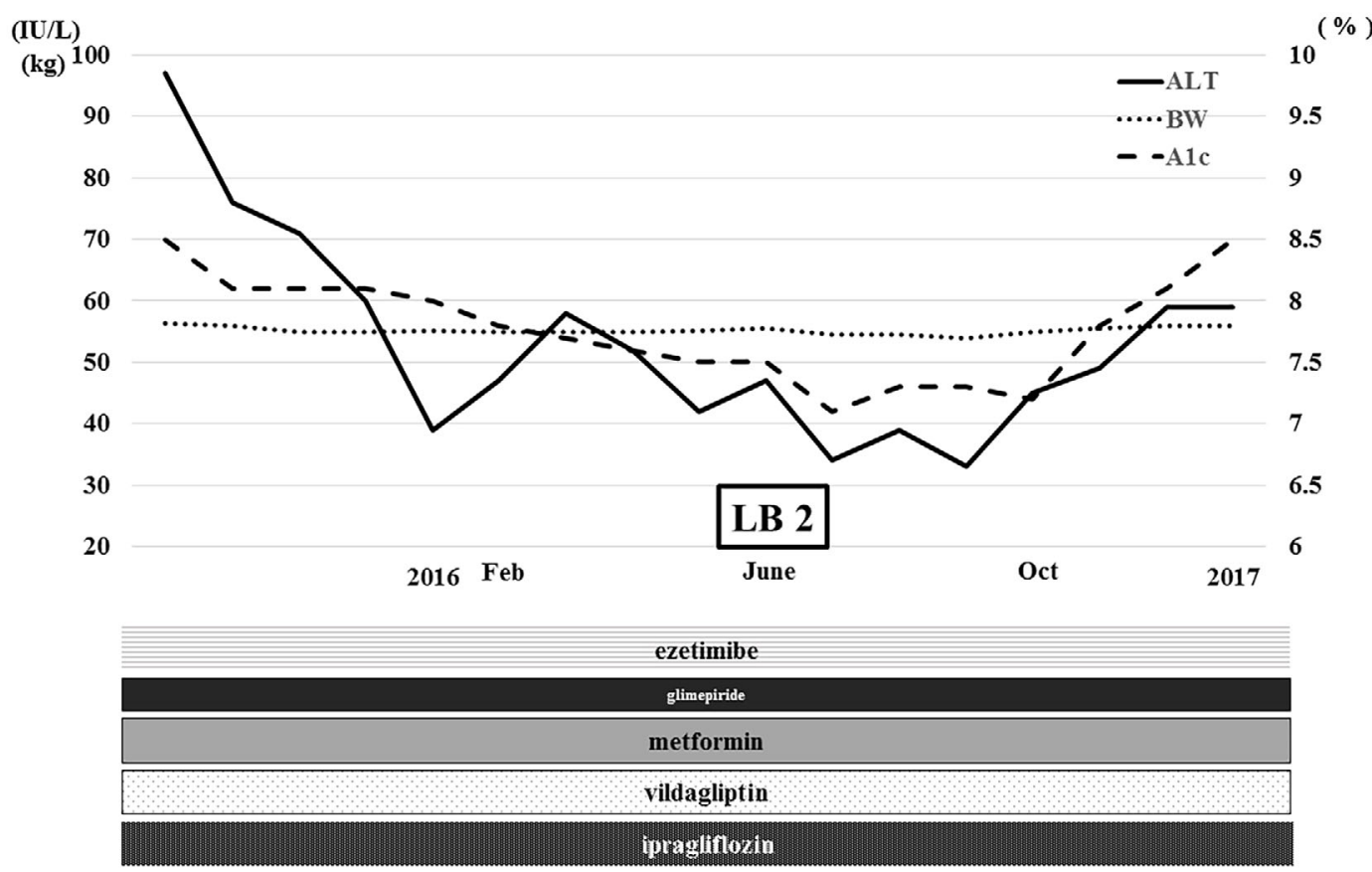

Figure 3. Clinical course after the administration of ipragliflozin. ALT: alanine aminotransferase, BW: body weight, A1c: glycated hemoglobin, LB: liver biopsy. Feb: February. Oct: October

Since we expected histological improvement in her liver, we obtained another liver sample with her consent in June 2016. This sample showed a marked improvement, especially in steatosis, inflammation and ballooning (Fig. 5), and her NAS was 3 (steatosis 1, inflammation 1, ballooning
1) (2). She continues to take ipragliflozin and has experienced no adverse effects yet (Fig. 3). 
(A)

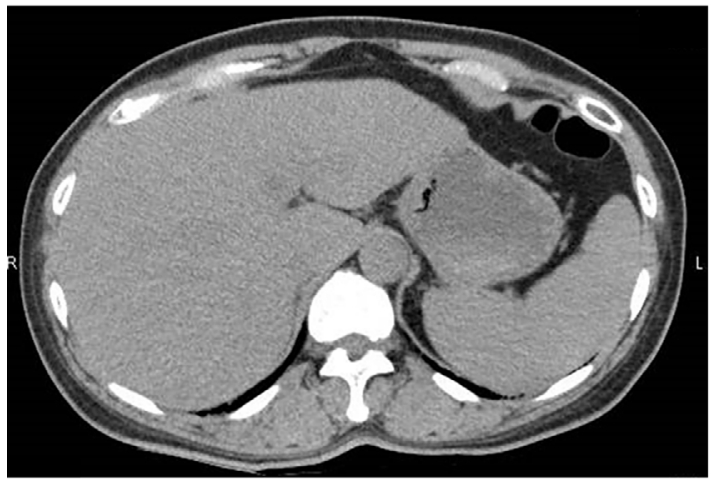

L/S ratio 1.02
(B)

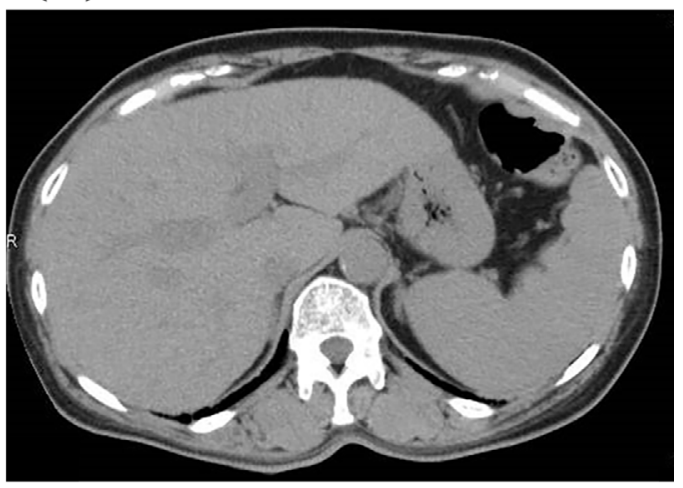

L/S ratio 1.10

Figure 4. Computed tomography of the liver. Before (A) and two months after the administration of ipragliflozin (B). L/S ratio: liver-to-spleen ratio

Table 2. Changes in Serum Fibrotic and Inflammatory Markers.

\begin{tabular}{ccccc}
\hline Serum marker & Unit & Normal range & Before & 4 months after \\
\hline Type IV collagen & $\mathrm{ng} / \mathrm{mL}$ & below 5.0 & 271 & 207 \\
Hyalronic acid & $\mathrm{ng} / \mathrm{mL}$ & $1.7-10.4$ & 596 & 517 \\
Ferritin & $\mathrm{ng} / \mathrm{mL}$ & $4.0-64.0$ & 132.4 & 38.6 \\
\hline
\end{tabular}

\section{Discussion}

This case suggested two important clinical issues. First, ipragliflozin can improve NASH not only clinically but also histologically. Second, the histological findings showed partial improvement of steatosis, inflammation, and ballooning, but not fibrosis.

\section{Clinical effects}

Before the administration of ipragliflozin, the patient had already taken three kinds of oral antidiabetic drugs and the lipid-lowering agent, ezetimibe. Her HbAlc level had been $7-9 \%$ for several years and was $8.5 \%$ just before the administration of ipragliflozin (Table 1, Fig. 1). After surgery in 2014, her liver data showed temporary improvement but worsened gradually again, and her HbAlc level increased to $8.5 \%$, probably because of overeating. She therefore started receiving ipragliflozin with reduced doses of glimepiride. After treatment for 4 months, her HbA1c level gradually decreased to $8 \%$, and her ALT level decreased dramatically to normal limits (Fig. 3). Her body weight also decreased to $55.2 \mathrm{~kg}(-1.4 \mathrm{~kg}$ from baseline). This clinical course strongly suggests that ipragliflozin was the greatest contributor to her improvement among the oral antidiabetic drugs administrated.

However, whether or not ipragliflozin was the greatest contributor to the body weight reduction is unclear, because she was receiving reduced doses of glimepiride and may have also improved her diet. Even though she was taking ipragliflozin continuously, her ALT level increased again be- cause of overeating in February 2016 (Fig. 3). However, in June 2016, when she was admitted to our hospital for a second liver biopsy, her ALT level had decreased to normal limits, and her HbAlc level had decreased to $7.1 \%$, probably because of diet modification. In October 2016, her ALT level and HbA1c level increased again, so it was believed that her diet had strongly contributed to her liver dysfunction. She resides in the Amami-Oshima area of Japan and prefers a diet of brown sugar, tropical fruits, and simple carbohydrates. We noticed again that diet therapy was very important, even after starting the administration of ipragliflrozin, for treating her NASH.

Some evidences supports the notions that SGLT2 inhibitors can improve the liver dysfunction of patients with type 2 diabetes (3-5). However, many studies have reported only laboratory findings, and histological findings including serum fibrotic markers, were not discussed. However, animal models of NASH with SGLT2 inhibitor have shown improvement of the histological findings (6-10), including with respect to fibrosis, inflammation, and ballooning.

In our case, improvements in the laboratory values, including findings for serum aminotransferases, inflammatory marker, and fibrotic markers, were concordant with the histological improvement. In cases where obtaining a liver sample is difficult, laboratory markers are useful for assessing the therapeutic effects in NASH patients with type 2 diabetes, as other groups indicated (11).

\section{Histological effects}

We performed a second liver biopsy nine months after starting the administration of ipragliflozin, because we ex- 


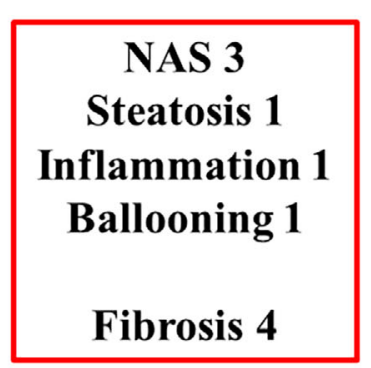

(b)
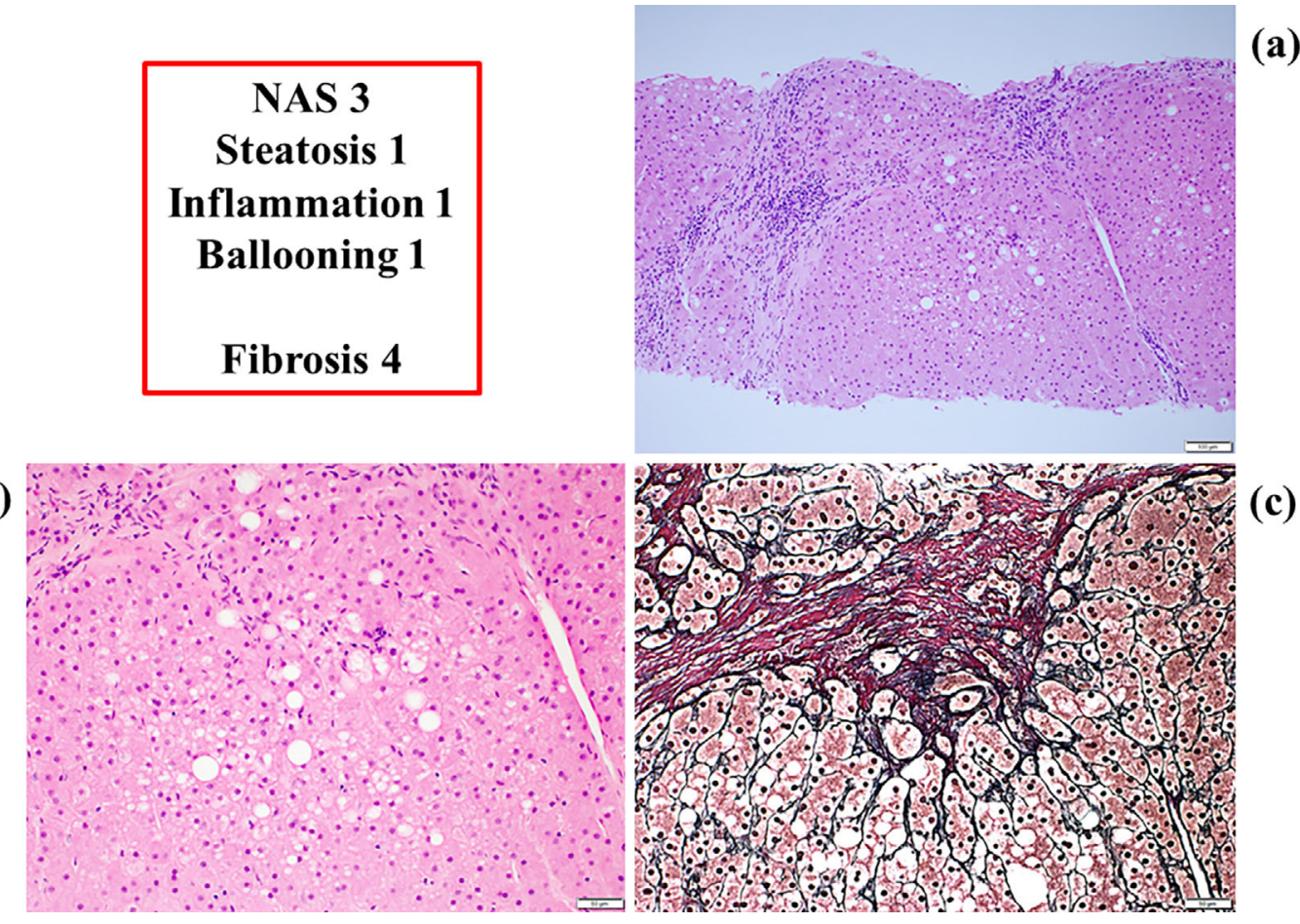

Figure 5. Histological findings of liver samples nine months after the administration of ipragliflozin. a: An overview of the liver sample [Hematoxylin and Eosin (H\&E) staining, 100×]. b: Fewer fat droplets and inflammatory neutrophil aggregations and less hepatocellular ballooning (H\&E staining, 200x). c: Interstitial fibrosis (silver staining, 200x). NAS: non-alcoholic fatty liver disease activity score

pected a dramatic histological improvement in her liver, particularly with regard to steatosis, inflammation, apoptosis, and fibrosis, based on her biochemical and imaging data. Her liver sample showed a marked improvement, especially in steatosis, inflammation, and ballooning (Fig. 5), and her NAS decreased from 6 to 3 . It was surprising that the total NAS decreased by 3 points after only 9 months of treatment. In the farnesoid $\mathrm{X}$ nuclear receptor ligand obeticholic acid for non-cirrhotic, non-alcoholic steatohepatitis (FLINT) trials (12), the obeticholic acid (OCA) group showed an average decrease of 1.7 points in the total NAS during 18 months of treatment. In the liraglutide safety and efficacy in patients with non-alcoholic steatohepatitis (LEAN) study (13), the liraglutide group showed an average decrease of 1.3 points in the total NAS during 12 months of treatment. Recently, in a 3-year study (14), 58\% of the pioglitazone group showed a decrease of $\geq 2$ points in the total NAS. In our case, the administration of ipragliflozin contributed to radical and quick histological improvement. As each drug has a different mechanism of reducing glucose levels, their effects on the histology are also different. We will discuss several mechanisms suspected of underlying the improvement in the histological findings.

The histological findings of liver samples before the administration of ipragliflozin showed accumulation of fat droplets mainly in the central vein area (zone 3), but even in the periportal area (zone 1). Since inflammatory neutrophil aggregations and hepatocellular balloonings were also found mainly in zone 3 , it reminded us that the accumulation of fat droplets leads to inflammation and cytotoxicity. However, the histological findings of liver samples after nine months of treatment showed fewer fat droplets, and inflammatory neutrophil aggregation and less hepatocellular ballooning in zone 3 , and almost none in zone 1 . Zone 1 is thought to be the most active area of $\beta$-oxidation (15), and these histological findings may indicate that the administration of ipragliflozin accelerated $\beta$-oxidation through caloric loss into urine. We speculated that a decrease in the number of fat droplets occurred first, subsequently leading to a decrease in the inflammation and cytotoxicity.

Recently, the pathogenesis of NASH/NAFLD has been understood through a multiple parallel hits theory (16), involving the effects of adipocytokines, gut microbiome, genetic factors, insulin resistance, lipotoxity, nutritional factors, oxidative stress, etc. We speculated that a decrease in the number of fat droplets led to a reduction in the insulin resistance, lipotoxity and oxidative stress by the administration of ipragliflozin (16). In this way, ipragliflozin may ameliorate NASH/NAFLD through a different mechanism from pioglitazone or liraglutide, so we can expect a synergistic effect with combined therapy of these agents (17).

\section{Points of regret}

The histological findings showed no improvement in fibrosis. This may be because her liver had already progressed to a cirrhotic state, or because the treatment duration was 
too short to show histological improvements in fibrosis. Although the serum fibrotic markers decreased to some degree, they never decreased to normal limits. The fibrosis score in NAS did not change, remaining 4 (Fig. 2, 5). The administration of ipragliflozin may be more effective in preventing fibrosis when administered at earlier stages of NASH/ NAFLD.

Since the fibrosis stage is reported to be independently associated with the long-term overall survival in NASH/ NAFLD (18), careful follow-up is needed in our case, even though the progression of NASH may have been stopped.

\section{Associations of Genetic Factors}

Recently, some groups have reported that patatin-like phospholipase domain containing 3 (PNPLA3) rs738409 (encoding the I148M variant) is associated with advanced fibrosis and hepatocellular carcinoma (HCC) (19). Therefore, we analyzed the PNPLA3 genotype using the patient's blood samples after receiving her informed consent, in accordance with the Declaration of Helsinki. In our case, the PNPLA3 genotype was $\mathrm{CG}$ and belonged to an intermediate-risk group. However, her platelet count had already decreased, and her liver fibrosis had progressed, and she is now at a high risk of developing HCC. This genetic background is concordant with the fact that her liver data were strongly affected by environmental factors, such as her diet (20).

In conclusion, we experienced a case of NASH with type 2 diabetes whose hepatic histological findings were improved by administration of the SGLT2 inhibitor, ipragliflozin. Ipragliflozin may be a useful therapeutic drug for NASH.

The authors state that they have no Conflict of Interest (COI).

\section{Acknowledgement}

We thank Dr. Sumida for performing the genetic analysis.

\section{References}

1. Yoneda M, Fujii H, Sumida Y, et al. Platelet count for predicting fibrosis in nonalcoholic fatty liver disease. J Gastroenterol 46: 1300-1306, 2011.

2. Kleiner DE, Brunt EM, Van Natta M, et al. Design and validation of a histological scoring system for nonalcoholic fatty liver disease. Hepatology 41: 1313-1321, 2005.

3. Cefalu WT, Leiter LA, Yoon K-H, et al. Efficacy and safety of canagliflozin versus glimepiride in patients with type 2 diabetes inadequately controlled with metformin (CANTATA-SU): 52 week results from a randomized, double-blind, phase 3 non-inferiority trial. Lancet 382: 941-950, 2013.

4. Ji L, Han P, Liu Y, et al. Canagliflozin in Asian patients with type 2 diabetes on metformin alone or metformin in combination with sulphonylurea. Diabetes Obes Metab 17: 23-31, 2015.

5. Leiter LA, Frost T, Polidori D, Balis DA, Xie J, Sha S. Effect of canagliflozin on liver function tests in patients with type 2 diabetes. Diabetes Metab 42: 25-32, 2016.

6. Hayashizaki-Someya Y, Kurosaki E, Takasu T, et al. Ipragliflozin, an SGLT2 inhibitor, exhibits a prophylactic effect on hepatic steatosis and fibrosis induced by choline-deficient L-amino aciddefined diet in rats. Eur J Pharmacol 754: 19-24, 2015.

7. Qiang S, Nakatsu Y, Seno Y, et al. Treatment with the SGLT2 inhibitor luseogliflozin improves nonalcoholic steatohepatitis in a rodent model with diabetes mellitus. Diabetol Metab Syndr 7: 104, 2015 .

8. Komiya C, Tsuchiya K, Shiba K, et al. Ipragliflozin improves hepatic steatosis in obese mice and liver dysfunction in type 2 diabetic patient irrespective of body weight reduction. PLoS One 11: e0151511, 2016.

9. Honda Y, Imajo K, Kato $\mathrm{T}$, et al. The selective SGLT2 inhibitor ipragliflozin has a therapeutic effect on nonalcoholic steatohepatitis in mice. PLoS One 11: e0146337, 2016.

10. Jojima T, Tomotsune T, Iijima T, Akimoto K, Suzuki K, Aso Y. Empagliflozin (an SGLT2 inhibitor), alone or in combination with linagliptin (a DPP-4 inhibitor), prevents steatohepatitis in a novel mouse model of non-alcoholic steatohepatitis and diabetes. Diabetol Metab Syndr 8: 45, 2016.

11. Sumida $\mathrm{Y}$, Yoneda $\mathrm{M}$, Hyogo $\mathrm{H}$, et al. A simple clinical scoring system using ferritin, fasting insulin, and type IV collagen $7 \mathrm{~S}$ for predicting steatohepatitis in nonalcoholic fatty liver disease. J Gastroenterol 46: 257-268, 2011.

12. Neuschwander-Tetri BA, Loomba R, Sanyal AJ, et al. Farnesoid X nuclear receptor ligand obeticholic acid for non-cirrhotic, nonalcoholic steatohepatitis (FLINT): a multicentre, randomized, placebo-controlled trial. Lancet 385: 956-965, 2015.

13. Armstrong MJ, Gaunt $P$, Aithal GP, et al. Liraglutide safety and efficacy in patients with non-alcoholic steatohepatitis (LEAN): a multicentre, double-blind, randomized, placebo-controlled phase 2 study. Lancet 387: 679-690, 2016.

14. Cusi K, Orsak B, Bril F, et al. Long-term pioglitazone treatment for patients with nonalcoholic steatohepatitis and prediabetes or type 2 diabetes mellitus: a randomized, controlled trial. Ann Intern Med 165: 305-315, 2016.

15. Tessari P, Coracina A, Cosma A, Tiengo A. Hepatic lipid metabolism and non-alcholoc liver disease. Nutr Metab Cardiovasc Dis 19: 291-302, 2009.

16. Tilg H, Moschen AR. Evolution of inflammation in nonalcoholic fatty liver disease: the multiple parallel hits hypothesis. Hepatology 53: 1836-1846, 2010.

17. Sumida Y, Seko Y, Yoneda M; Japan Study Group of NAFLD (JSG-NAFLD). Novel antidiabetic medications for nonalcoholic fatty liver disease with type 2 diabetes mellitus. Hepatol Res 47: 266-280, 2017.

18. Angulo P, Kleiner DE, Dam-Larsen $S$, et al. Liver fibrosis, but no other histologic features, is associated with long-term outcomes of patients with nonalcoholic fatty liver disease. Gastroenterology 149: 389-397, 2015.

19. Seko Y, Sumida Y, Tanaka S, et al. Development of hepatocellular carcinoma in Japanese patients with biopsy-proven non-alcoholic fatty liver disease: Association between PNPLA3 genotype and hepatocarcinogenesis/fibrosis progression. Hepatol Res 2016 (Epub ahead of print).

20. Honda Y, Yoneda M, Kessoku T, et al. Characteristics of nonobese non-alcoholic fatty liver disease: effect of genetic and environmental factors. Hepatol Res 46: 1011-1018, 2016.

The Internal Medicine is an Open Access article distributed under the Creative Commons Attribution-NonCommercial-NoDerivatives 4.0 International License. To view the details of this license, please visit (https://creativecommons.org/licenses/ by-nc-nd/4.0/).

(C) 2017 The Japanese Society of Internal Medicine

Intern Med 56: 2739-2744, 2017 